\title{
Motivação para Mudança no Uso de Substâncias entre Usuários de Drogas Encaminhados pela Justiça
}

\author{
Itamar José Félix Junior ${ }^{*}, 1$ \\ Orcid.org/0000-0001-6350-5194 \\ Paulo Renato Vitória Calheiros ${ }^{1}$ \\ Orcid.org/0000-0003-1897-4180 \\ Pedro di Tárique Barreto Crispim ${ }^{1}$ \\ Orcid.org/0000-0003-1243-348X
}

${ }^{1}$ Universidade Federal de Rondônia, Porto Velho, RO, Brasil

\section{Resumo}

O projeto Justiça Terapêutica, que encaminha para tratamento pessoas com dependência de substâncias psicoativas que cometeram crimes, tem ganhado destaque no Brasil. Este estudo teve como objetivo avaliar os estágios de motivação em pessoas encaminhadas pela justiça para tratamento de dependência de substâncias psicotrópicas. A amostra foi constituída por 120 pessoas que estavam em tratamento CAPSAD e internados em Comunidades Terapêuticas, na cidade de Porto Velho, Rondônia. Os indivíduos foram alocados em dois grupos para comparação da motivação para mudança de comportamento: um formado por pessoas encaminhadas pela justiça (Grupo Justiça) e o outro constituído por pessoas que estavam em tratamento por qualquer outro motivo, exceto encaminhamento judicial (Grupo Outros). Foram utilizados para avaliar as variáveis de motivação dos grupos a Escala URICA para substâncias Ilícitas, Régua de Prontidão e Prontidão para Mudança. Os resultados demonstraram que não foram encontradas diferenças estatisticamente significativas na motivação das pessoas encaminhadas pela justiça e as pessoas encaminhadas pelas formas de indicação mais usuais para tratamento. Ressalta-se a importância de avaliar a motivação em todas as fases do tratamento, em vista da melhor adesão e sucesso terapêutico das pessoas com problemas com a justiça e com o uso de substâncias.

Palavras-chave: Justiça Terapêutica, motivação para mudança de comportamento, tratamento dependência de drogas.

\section{Motivation for Change in Substances Use among Drug Users Referred by the Justice System}

\begin{abstract}
The Therapeutic Justice project, which refers people with psychoactive substance dependence who committed crimes for treatment, has gained prominence in Brazil. This study aimed to evaluate the

* Endereço para correspondência: Av. Costa e Silva, 2241, Centro, Itapuã do Oeste, RO, Brasil 76861-000. Fone: 69 99233-0632. E-mail: itamar.junior44@hotmail.com

Nota do autor: Estudo desenvolvido em projeto de mestrado acadêmico de Psicologia, sem fomento.

Apoio: CAPES, Comunidades Terapêuticas, Centro de Atenção Psicossocial-Álcool e Drogas (CAPS-AD), Mestrado Acadêmico em Psicologia da Fundação Universidade Federal de Rondônia (MAPSI-UNIR).
\end{abstract}


stages of motivation in people referred by the justice system for the treatment of psychotropic substance dependence. The sample consisted of 120 people who were being treated in CAPS-AD and Therapeutic Communities in the city of Porto Velho, Rondônia, Brazil. The individuals were divided into two groups, one formed by people referred by the justice system (Justice System Group) and another made up of people who were being treated for any other reason (Others Group), to compare the motivation to change behavior between the groups. The URICA Scale for Illicit Substances, the Readiness Ruler and Readiness for Change were used to evaluate the motivation variables of the groups. The results showed that there were no statistically significant differences in motivation between the people referred by the justice system and the people with other more usual forms of indication for treatment. The importance of assessing the motivation during all stages of treatment is emphasized, aiming for better adherence and therapeutic success of people with problems with the law and the use of substances.

Keywords: Therapeutic Justice, motivation to change behavior, drug addiction, treatment.

\section{Motivación para el Cambio de la Conducta Adictiva entre Consumidores de Drogas Enviadas por la Justicia}

\section{Resumen}

El proyecto Justicia Terapéutica hacia delante para tratar a personas con dependencia de sustancias psicoactivas que cometieron crímenes ha ganado importancia en Brasil. Este estudio tuvo como objetivo evaluar las etapas de la motivación en las personas enviadas por la justicia para el tratamiento de la dependencia de sustâncias psicotrópicas. La muestra fue de 120 personas que estaban siendo tratados en el CAPS-AD y em Comunidades Terapéuticas en la ciudad de Porto Velho, Rondonia. Los individuos fueron divididos en dos grupos, uno formado por personas enviadas por la justicia (Grupo de Justicia) y otro formado por personas que estaban siendo tratados por cualquier otra razón, excepto la remisión judicial (otro grupo), para comparar entre los grupos motivación para cambiar comportamiento. Se utilizó para evaluar las variables de motivación de los grupos URICA Escala ilícito Regla de Preparación y disposición para el cambio. Los resultados mostraron que hubo diferencias estadísticamente significativas en la motivación de las personas enviadas por la justicia y las personas enviadas por las formas de indicación es más tratamientos habituales. Hacemos hincapié en la importancia de evaluar la motivación en todas las etapas del tratamiento, con el fin de una mejor adherencia y el éxito terapéutico de las personas con problemas con la ley y el uso de sustancias.

Palabras clave: Justicia Terapéutica, motivación para el cambio de comportamiento, tratamientos para la drogadicción.

O Brasil enfrenta uma grave crise penitenciária, na qual o número de detentos aumenta a cada ano, a despeito do número de vagas nos presídios. Atualmente o país possui a quarta maior população carcerária, com mais de 600 mil presos. Desses, $41 \%$ são presos provisórios e $27 \%$ presos por tráfico de drogas (Ministério da Justiça, 2015). A grande maioria dessa população necessita de cuidados à saúde, motivados por muitas enfermidades, como as DST's e a dependência por drogas. Segundo Soares e Bueno (2016), o atendimento à saúde dos presidiários é deficitário e é só mais uma das problemáticas do sistema carcerário. Contudo, a superlotação é considerada o maior problema e as condições das prisões são insalubres e perigosas para a saúde humana.

Algumas medidas têm sido tomadas para diminuir a superlotação. Entre as alternativas encontradas destacam-se as audiências de custódia e a utilização de tecnologia, como as tornozeleiras eletrônicas (Soares \& Bueno, 2016). Contudo um projeto visando também diminuir a superlotação objetivava o tratamento do uso 
de drogas e a diminuição da reincidência da criminalidade. O projeto denominado "Justiça Terapêutica" surgiu pela iniciativa de promotores de justiça do Rio Grande do Sul motivados pela crescente problemática que envolvia usuários de drogas e a prática de crimes.

Aliado a isso, em 2006 foi promulgada a Lei $\mathrm{n}^{\circ} 11.343$, instituindo o Sistema Nacional de Políticas Públicas sobre Drogas (SISNAD), na qual foram incluídos, pela primeira vez, alguns artigos que abordam o tratamento para indivíduos com problemas associados ao uso de drogas que tiveram problemas com a justiça. Com isso, houve a abertura para que o encaminhamento de pessoas envolvidas com crimes e problemas com drogas fossem encaminhados para tratamento. Geralmente pessoas que cometeram crimes e são usuários de drogas possuem problemas com mais de uma droga e possuem vulnerabilidades que dificultam o acesso e ao término de um tratamento. Desse modo, se configura como fundamental a motivação para alcançar o sucesso terapêutico, principalmente no que se refere a tratamentos impostos por força maior. Estudos com adultos demonstraram que a motivação é um fator importante na busca, adesão e desfecho positivo de intervenções terapêuticas para usuários de drogas (Prochaska, DiClemente, \& Norcross, 1992). A motivação, portanto, é um dos componentes primordiais para o tratamento de da dependência de drogas.

As abordagens motivacionais são vistas como fundamentais no tratamento de usuários de drogas, principalmente na adesão. Entre as abordagens se destaca o Modelo Transteórico de Motivação para a Mudança (MTT) que iniciou estudos acerca da mudança de comportamentos vistos como problemáticos e que muitas vezes são caracterizados pela dificuldade no tratamento ou na adesão dele (Prochaska, 2013). A premissa é que a mudança se dá em um processo dividido em diferentes estágios até alcançar a efetiva mudança, sendo cinco estágios, flexíveis entre si. Tais estágios são: Pré-contemplação, Contemplação, Preparação, Ação e Manutenção. A Pré-contemplação se caracteriza como estágio no qual a pessoa não enxerga que há problemas e não considera a mudança. Nesse estágio, a pes- soa comumente não se convence que seu padrão de comportamento está lhe trazendo transtornos e assim dificilmente está disposta a considerar a mudança (Oliveira, Ludwig, Freire, \& Zanetello, 2012). O estágio da Contemplação é onde se configura o sentimento de ambivalência entre querer mudar ou manter o comportamento. A pessoa começa a pensar na possibilidade de modificar seu comportamento. A Preparação é o estágio seguinte à Contemplação, uma vez que o indivíduo possa ter encontrado motivos para mudar e começa a planejar a mudança, firmando um "compromisso" no tratamento. A Ação é o estágio em que se caracteriza pelas estratégias escolhidas colocadas em prática. No estágio da Manutenção, o indivíduo trabalha e consolida os ganhos obtidos na Ação e previne a recaída.

Impulsionados pela interferência da motivação no tratamento, Polaschek, Anstiss e Wilson (2010) compararam algoritmos de uma escala denominada Criminogenic Needs Inventory's e da escala URICA, visando à validação das propriedades psicométricas da escala em 260 prisioneiros, na avaliação da motivação de mudança do comportamento criminoso. Os resultados indicaram suporte preliminar para a confiabilidade e validade da URICA na avaliação do estágio de mudança relacionadas a comportamentos criminosos com presos do sexo masculino. Já o estudo de El-Bassel et al. (1998) visou avaliar a qualidade psicométrica da Escala URICA e o estágio de motivação em uma população de mulheres encarceradas. A amostra foi dividida em cinco grupos: negação; não envolvidas; ambivalente; tomada de decisão e participação. Os resultados mostraram que o grupo "negação" apresentou escores acima da média na subescala Pré-contemplação, sendo que, por outro lado, o grupo "ambivalência" pontuou acima da média em todas subescalas.

No Brasil, Oliveira, Szupszynski e DiClemente (2010) fizeram um estudo em que avaliaram o estágio motivacional e o relacionaram com a adesão ao tratamento em 103 adolescentes infratores usuários de substâncias ilícitas que estavam em cumprimento de medidas socioeducativas. Os resultados indicaram que $66 \%$ dos adolescentes não aderiram ao tratamento e 
69,3\% estavam no estágio de Pré-contemplação no momento da avaliação inicial. Ainda foi possível relacionar a presença do Transtorno de Conduta e adesão, onde se obteve relação significativa evidenciando que quem possuía o transtorno tinha menor probabilidade de aderir ao tratamento.

Acerca de intervenções voltadas a pessoas com envolvimento judicial, uma pesquisa realizada pelo National Institute on Drug Abuse (NIDA), dos EUA, concluiu que o modelo de tratamento compulsório é tão eficiente quanto o voluntário, e que as pessoas encaminhadas compulsoriamente estavam menos motivadas somente no início do tratamento, mas com o passar do tempo passaram a refletir sobre si mesmos e a reconhecer a necessidade do tratamento (Kelly, Finney, \& Moos, 2005). Uma análise que revisou os resultados de 50 estudos que incluíam modelos experimentais e quase experimentais em presos com problemas com drogas indicou que estes são menos propensos a reincidir, comparado com presos que não passam por alguma intervenção sobre drogas (Wilson, Mitchell, \& MacKenzie, 2006).

Estudo mais recente de avaliação da motivação demonstrou que o suporte parental no tratamento para uso de drogas prediz maior motivação em adolescentes, levando-os a dar mais importância ao tratamento (Serafini, Stewart, Wendt, \& Donovan, 2017). Assim, a mudança entre pessoas encaminhadas pela justiça e também entre adolescentes usuários de drogas se constituem um desafio para terapeutas e instituições, na qual a rede de apoio se mostra como um importante adjuvante no tratamento.

Ademais, os prejuízos ocasionados pelo uso de drogas acabam aumentando a vulnerabilidade social do indivíduo ou de um grupo, podendo ocorrer o contrário disso: a vulnerabilidade social aumentar o consumo de drogas. Logo, esses aspectos podem determinar maiores riscos desses indivíduos se envolverem em atitudes conflitivas com a lei. Todos esses fatores podem estar interligados e, portanto, essa relação entre crimes e uso de drogas deve ser mais bem estudada no Brasil, principalmente em relação à oferta de tratamento para usuários de drogas e motivação dentro do sistema de justiça criminal.

Com tais panoramas, o presente estudo partiu da hipótese de que a motivação de pessoas encaminhadas pela justiça seria diferente de pessoas encaminhadas para tratamento por outros fatores, devido à crença de que o sujeito encaminhado usaria o tratamento como um motivo para não ficar preso e, assim, não levaria o tratamento a sério, ou não estaria tão motivado para tal. Assim, considerando que o encaminhamento judicial de pacientes para tratamento por dependência e uso de drogas tem sido uma prática que vem ganhando destaque no Brasil, além da necessidade de se obter informações sobre esse tema, este estudo teve por objetivo avaliar o estágio de motivação para mudança em pacientes encaminhados pela Justiça para tratamento para dependência de drogas em Comunidades Terapêuticas e no Centro de Atenção Psicossocial Álcool e outras Drogas (CAPS-AD) de Porto Velho/RO.

\section{Método}

Trata-se de um estudo transversal, descritivo, de comparação entre dois grupos (Justiça e Outros), sendo utilizada a metodologia quantitativa.

\section{Amostra}

No total, a amostra foi constituída de 120 participantes. A seleção da amostra foi por conveniência e a escolha das instituições se deu de acordo com o local em que os participantes encaminhados pela justiça estavam se tratando. A Vara de Execução de Penas e Medidas Alternativas (VEPEMA) forneceu uma lista com todas as instituições cadastradas e com o nome dos encaminhados. O critério de inclusão foi ter acima de 18 anos, não ter feito uso de drogas nas últimas 24 horas, concordar em participar da pesquisa e não ter desistido do tratamento, no caso dos encaminhados. A pessoa encaminhada que descumpre ou não adere ao tratamento, desistindo, tem o risco de voltar a ser preso ou cumprir outra medida alternativa, como a Prestação de Servi- 
ços à Comunidade. Já os critérios de exclusão da pesquisa para os dois grupos foram: fazer menos de 24 pontos no Mini-Exame de Estado Mental (MEEM) e apresentar sintomas ou síndromes psicóticas.

Os participantes da pesquisa foram encontrados em dois modelos de tratamento ofertados no estado de Rondônia: a internação em instituições que se autodenominavam Comunidades Terapêuticas (CTs) correspondeu a 74,2\% $(n=89)$ e $25,8 \%(n=31)$ em modelo ambulatorial realizado no CAPS-AD. Para análise comparativa, dividiram-se os participantes em dois grupos: "Grupo Justiça", formado por pessoas encaminhadas pela Justiça para o tratamento e o "Grupo Outros", constituído por pessoas que estavam se tratando por diversos outros motivos, que não seja o encaminhamento judicial. Houve cinco recusas $(4,16 \%)$ de participação, sendo três por pessoas do Grupo Justiça.

\section{Local}

A pesquisa foi realizada em Comunidades Terapêuticas e no CAPS-AD da cidade de Porto Velho. Ao todo, nove instituições foram visitadas. A maioria das CTs são filantrópicas e mantidas por doações, com exceção de três que possuem leitos financiados pela Secretaria Nacional de Política sobre Drogas (SENAD) e pelo Governo do Estado.

\section{Instrumentos}

Mini-Exame do Estado Mental (MEEM): trata-se de um teste amplamente utilizado em ambiente clínico para o rastreamento de quadros demenciais. O MEEM foi traduzido para a língua portuguesa por Bertolucci, Brucki, Campacci e Juliano (1994).

URICA para Ilícitas (Rhode Island Change Assessment Questionnaire): A URICA para substâncias Ilícitas (versão reduzida) foi validada por Szupszynski e Oliveira (2008) a partir de um estudo com usuários de substâncias psicotrópicas ilícitas. A análise de consistência interna da validação foi satisfatória $(\alpha=0,657)$. $\mathrm{O}$ instrumento tem 24 itens subdivididos em quatro subescalas: Pré-contemplação (6 itens);
Contemplação (6 itens); Ação (6 itens) e Manutenção (6 itens).

Régua de Prontidão: Consiste em uma régua numerada de 0 a 10 a qual o pesquisador perguntará ao paciente em qual ponto da régua melhor reflete o quão pronto ele está, no presente momento, para mudar seu comportamento (Velasquez, Maurer, Crouch, \& DiClemente, 2001).

Entrevista Estruturada: Foi composta por itens abertos e fechados que abordam as seguintes variáveis: (a) características sociodemográficas (idade, gênero, estado civil, nível de escolaridade, profissão e ocupação); (b) características de elegibilidade (critérios de inclusão e exclusão); (c) padrão de uso de drogas, problemas associados; (d) tratamentos anteriores, vida pregressa, uso de medicamentos e; (e) socioeconômicas, avaliados pelo Critério de Avaliação Econômica Brasil 2015 (Associação Brasileira de Empresas de Pesquisa [ABEP], 2015).

\section{Procedimentos de Coleta de Dados}

Os procedimentos de coleta de dados seguiram as especificidades do encaminhamento judicial para o tratamento, na qual a Vara de Execução de Penas e Medidas Alternativas (VEPEMA), através de uma equipe de profissionais composta por psicólogos e assistentes sociais responsáveis pela aplicação do projeto piloto "Justiça Terapêutica" do Tribunal de Justiça de Rondônia (TJRO), encaminha indivíduos para o tratamento para dependência química no local de sua preferência, após análise e decisão do Juiz. O encaminhamento também pode ocorrer antes do término da análise processual, quando o sujeito solicita o encaminhamento através de ação judicial. Apesar do encaminhamento não ser coercitivo ou involuntário, o indivíduo que não adere ao tratamento ou o abandona, pode ter esse direito retirado e assim, então, voltar a cumprir a sua pena em regime fechado. Buscou-se entrevistar todos os sujeitos que foram encaminhados.

A coleta de dados foi realizada por auxiliares de pesquisa, estudantes de psicologia que desconheciam os objetivos do estudo e foram previamente treinados na aplicação dos instrumentos da pesquisa. Os procedimentos de esco- 
lha dos participantes se deram da seguinte maneira: nas $\mathrm{CTs}$, os participantes que não eram encaminhados pela justiça (Grupo Outros) foram escolhidos a partir de sorteio de uma lista de todos os internos do local, onde eram escolhidos os de números par. A coleta de dados no CAPS-AD foi realizada todas as quartas feiras (dias de grupoterapia) e em alguns dias com consultas médicas, por conta de serem dias com mais pacientes frequentando a instituição. Nesses dias, os indivíduos convidados a participar da pesquisa foram os definidos pelo número par da lista de espera para participar dos grupos ou consultas médicas.

\section{Procedimentos Éticos}

O estudo obteve aprovação do Comitê de Ética em Pesquisa da Universidade Federal de Rondônia, sob o parecer $\mathrm{n}^{\circ}$ 1.205.918 (CAAE: 44845215.1.0000.5300). Todos aqueles que concordaram em participar assinaram o Termo de Consentimento Livre e Esclarecido, de acordo com a Resolução No 466/12 do Conselho Nacional de Saúde. Todos responderam à pesquisa em locais apropriados, na própria instituição, individualmente, em sessões com duração média de 25 minutos, durante o período de Outubro de 2015 a Março de 2016.

\section{Análise dos Dados}

As informações coletadas foram processadas em uma planilha eletrônica no programa Statistical Package for the Social Sciences (SPSS), versão 20.0. As variáveis quantitativas e ordinais foram descritas através dos valores mínimo (Mín), primeiro quartil $\left(Q_{1}\right)$, mediana $(M e d)$, terceiro quartil $\left(Q_{3}\right)$ máximo (Máx), média $(X)$ e desvio padrão $(D P)$. As variáveis nominais foram descritas através de suas frequências absolutas e percentagens. Para testar a normalidade das distribuições foi aplicado o teste de Kolmogorov-Sminov e para a comparação dos grupos foi verificada a partir do teste não paramétrico de Mann-Whitney. A comparação entre as proporções das variáveis nominais foi verificada através do teste Qui-Quadrado. O nível de significância adotado foi de $5 \%$.

\section{Resultados}

A idade média $( \pm D P)$ da amostra foi de 35,21 anos ( $\pm 11,06$ anos). As descrições relativas às características sociodemográficas estão incluídas na Tabela 1. Buscou-se comparar dois grupos de pessoas em tratamento para avaliação do estágio de motivação: o "Grupo Outros" foi composto por $70,8 \%(n=85)$ da amostra e o "Grupo Justiça", que foi composto por 29,2\% $(n=35)$ da amostra.

A respeito da forma de encaminhamento para o tratamento dos participantes do Grupo Outros: $40,9 \%(n=38)$ relataram que estavam se tratando "por vontade própria", sem indicação alguma; 30\% $(n=28)$ tinham sido encaminhados pela família, $12,9 \%(n=12)$ tiveram ajuda de amigos, $8,6 \%(n=8)$ foram indicados pelo trabalho, $4,3 \%(n=4)$ tiveram ajuda de instituições ou serviços comunitários, como igrejas ou abrigos e, por fim, 3,3\% $(n=3)$ foram encaminhadas por instituições de saúde ou de assistência social.

Outro ponto de destaque foram os problemas com uso de drogas e crimes: na amostra total $56,7 \%$ afirmaram terem cometido delitos para obter drogas. Acerca dos crimes entre os participantes do Grupo Justiça $(n=35), 42,9 \%$ $(n=15)$ tiveram problemas com a justiça por conta do tráfico de drogas, 25,7\% $(n=9)$ por roubo, $11,4 \%(n=4)$ por furto, $11,4 \%(n=4)$ estavam respondendo pelo crime de violência doméstica, $5,7 \%(n=2)$ por direção perigosa e $2,9 \%(n=1)$ por agressão.

Em relação ao uso de drogas que levaram os participantes a procurar tratamento: a maioria usava o crack (50\%); em seguida a cocaína (22,5\%) e o álcool (20\%), e por fim a maconha $\left(7,5 \%\left[\chi^{2}=46,200, g l=3, p<0,0001\right]\right)$. O tempo mediano de dias em tratamento foi de 102,50 dias $\left(M i n=1,0 ; Q_{1}=53,75 ; Q_{3}=180,00 ;\right.$ Máx $=3650, \quad X=142,56, D P=411,06)$. Já o tempo mediano de dias em abstinência foi de 90 dias (Min=1,0; $Q_{1}=30,50 ;$ Med $=90,00 ; Q_{3}=180,00$; Má $x=1825,0 ; \quad X=142,56 ; D P=220,28)$. Acerca dos grupos: o tempo mediano em tratamento do Grupo Outros foi de 96,00 dias e a do Grupo Justiça foi de 75,00 dias. O tempo mediano de abstinência do uso de drogas do Grupo 
Tabela 1

Descrição dos Dados Sóciodemográficos da Amostra

\begin{tabular}{cccccc}
\hline Variável & $n$ & $\%$ & $\chi^{2}$ & valor- $p$ \\
\hline
\end{tabular}

Sexo

Masculino

Feminino

Cor da pele

Parda

Branca

Preta

Estado civil

Solteiro(a):

Casado(a)/mora com parceira(o):

Separado(a)/divorciado(a):

Viúvo(a)

Possui Filhos

Não

$\operatorname{Sim}$

Grau de escolaridade

Ensino fundamental incompleto

Ensino médio completo:

Ensino médio incompleto

Superior incompleto

Ensino fundamental completo

Não sabe ler nem escrever

Classe social
A
B
C

D-E

Situação de emprego atual

Desempregado
Empregado
Trabalho informal/autônomo
Aposentado

Cometeu delitos para comprar drogas

Sim

Não
103

17

$85,8 \%$

$14,2 \%$

$60,208<0,0001$

74

36

10

$61,7 \%$

$30 \%$

$51,800<0,0001$

$8,3 \%$

80

$66,7 \%$

30

09

$25 \%$

$7,5 \%$

$126,067<0,0001$

01

$0,8 \%$

71

49

$59,2 \%$

$40,8 \%$

3,675

0,0552

$47,5 \%$

22

$18,3 \%$

20

11

$16,7 \%$

$9,2 \%$

$96,800<0,0001$

09

$7,5 \%$

01

$0,8 \%$

07

$5,8 \%$

$61,7 \%$ 
Já usou drogas no trabalho

$\begin{array}{lllll}\text { Sim } & 67 & 55,8 \% & 1,408 & 0,2353 \\ \text { Não } & 53 & 44,2 \% & & \end{array}$

Tentou parar sozinho e não conseguiu

Sim

Não

Passou noite na rua nos últimos seis meses

Sim

Não

$100 \quad 83,3 \%$

20
$52,008<0,0001$

$33 \quad 27,5 \%$

87
$72,5 \%$
23,408 $<0,0001$
Outros foi de 90,00 dias e a do Grupo Justiça foi de 75,00 dias. Importante também ressaltar o tempo mediano em abstinência entre os modelos de tratamento, que foi de 97,00 dias para os sujeitos que estavam se tratando em CTs (Min=0 dias; $Q 1=60,00 ; Q 3=180,00 ;$ Má $=1825$ dias; $X=139,66 ; D P=207,63)$, contra 30,00 dias dos participantes do modelo ambulatorial CAPS-AD ( $M i n=0$ dias; $Q 1=1,00 ; Q 3=210,00$; Má $x=910$ dias; $X=150,90 ; D P=256,76$ ).

Do total de participantes, $23,3 \%(n=28)$ não pensavam em se tratar, dos quais $35,7 \%(n=10)$ eram do Grupo Justiça. O tempo mediano no qual o sujeito passou a considerar o tratamento foi de 30,00 dias, não havendo diferenças estatisticamente significativas entre os grupos Justiça $(\mathrm{Med}=9,00$ dias; $M i n=0$ dias, $Q 1=$ $0, Q 3=97,50$, Má $=440$ dias, $X=72,47, D P=$ 120,36) e Outros (Med=30,00 dias; $M i n=0$ dias; $Q=1,00 ; Q 3=90,00 ;$ Má $x=5000$ dias; $X=171,46$; $D P=575,23$ ), na comparação não houve diferença estatísticas significativas ( $U$ de Mann Whitney $=1120,50 ; p=0,111)$.

A respeito das variáveis de motivação, os dados da amostra total da Régua de Prontidão, Escala URICA para Ilícitas e Prontidão para Mudança estão descritos na Tabela 2.

Tabela 2

Descrição da Régua de Prontidão, Escala URICA e Prontidão para Mudança

\begin{tabular}{lccccccccc}
\hline \multicolumn{1}{c}{ Variável } & $N$ & Min & $Q_{1}$ & Med & $Q_{3}$ & Máx & $\chi$ & $D P$ & \\
\hline Régua de prontidão & $118^{*}$ & 0,00 & 5,00 & 8,00 & 9,00 & 10,00 & 7,07 & 2,74 & \\
Pré-contemplação & 120 & 6,00 & 13,00 & 15,00 & 18,00 & 26,00 & 15,30 & 3,72 & 2,45 \\
Contemplação & 120 & 16,00 & 13,00 & 24,00 & 25,00 & 30,00 & 23,54 & 2,45 \\
Ação & 120 & 14,00 & 24,00 & 26,00 & 28,00 & 30,00 & 25,72 & 3,03 & 3,33 \\
Manutenção & 120 & 14,00 & 21,25 & 23,00 & 25,00 & 30,00 & 23,13 & 3,06 \\
Prontidão para mudança & 120 & 36,00 & 52,00 & 55,00 & 63,00 & 76,00 & 57,08 & 8,06 \\
\hline
\end{tabular}

Nota. Min: mínimo; Med: mediana; Máx: máximo; $X$ : média; $D P$ : desvio padrão.

*Duas pessoas não sabiam e/ou não quiseram responder, sendo uma do Grupo Outros e um do Grupo Justiça.

Acerca da consistência interna da Escala URICA para Ilícitas, a fidedignidade baseada no coeficiente de Cronbach foi de $\alpha=0,684$, apresentando nível correspondente ao de validação do instrumento (Szupszynski \& Oliveira, 2008). O nível de Prontidão para
Mudança foi obtido a partir da soma dos escores da Contemplação, Ação e Manutenção, subtraindose a Pré-contemplação, sendo, portanto, colocada da seguinte maneira: Contemplação $(\mathrm{C})+$ Ação (A) + Manutenção (M) - Pré-contemplação (PC) $=$ prontidão total $(\mathrm{PT})$. Os dados de comparação 
entre o Grupo Outros e o Grupo Justiça, com valores mínimos, mediana, máximo, média e desvio padrão das subescalas da URICA para Ilícitas, Régua de Prontidão e da Prontidão para Mudança estão demonstrados na Tabela 3.

Os dados comparativos entre o Grupo Justiça e o Grupo Outros em relação ao modelo de tratamento Ambulatorial (CAPS-AD) e o modelo de Internação (CTs) da Régua de Prontidão, das subescalas da Escala Urica e Prontidão para Mudança estão apresentados na Tabela 4.

Foram feitas perguntas direcionadas para os participantes do Grupo Justiça $(\mathrm{n}=35)$, na qual $88,6 \%(n=31)$ estavam presos antes de receberem a medida alternativa de tratamento para dependência química; 45,7\% $\quad(n=16)$ estavam se tratando por conta do uso do crack; $28,6 \%(n=10)$ por uso de cocaína; $14,3 \%(n=5)$ por maconha e $11,4 \%(n=4)$ por álcool. Acerca do número de vezes que a pessoa procurou tratamento antes do encaminhamento: $34,3 \%$ $(n=12)$ estavam se tratando pela primeira vez, outras $31,4 \% \quad(n=11)$ estavam no segundo tratamento e outros 34,3\% $(n=12)$ já tinham se tratado três ou mais vezes. Houve diferença estatisticamente significativa entre os tempos medianos fazendo uso problemático de substâncias entre o Grupo Justiça $(\mathrm{Med}=7$ anos; $M i n=0$ ano, $Q 1=3,00 ; Q 3=10,00$; $M a ́ x=22$ anos; $X=7,9$ anos; $D P=5,73$ anos) e o Grupo Outros (Med=10 anos; $M i n=1$ ano; $Q 1=5 ; Q 3=16,50$; Máx $=40$ anos; $X=12,00$ anos; $D P=8,94$ anos), havendo diferenças entre os grupos ( $U$ de MannWhitney $=1074,50 ; p=0,021$ ).

\section{Discussão}

O estudo apresentou resultados interessantes no que tange à motivação dos encaminhados pela justiça, em vista da hipótese inicial. De acordo com a Escala URICA para Substância Ilícitas, foi demonstrado que a motivação de pessoas encaminhadas pela justiça não difere de pessoas encaminhadas para tratamento por outras fontes, como pela família ou por si próprio. Entretanto foram percebidas diferenças entre os grupos quanto à localização no estágio de motivação para mudança de comportamento. Além disso, os resultados da amostra total são semelhantes aos obtidos no estudo de validação da escala URICA para Substâncias Ilícitas (Szupszynski \& Oliveira, 2008).

A divisão entre grupos objetivou analisar melhor as diferenças possíveis entre os dois grupos com encaminhamentos diferentes para o tratamento, verificando-se não haver diferenças estatísticas entre os grupos no que tangem às variáveis de motivação. Contudo, o estudo de Prendergast, Greenwell, Farabee e Hser (2009) demonstrou que presidiários em tratamento obtiveram médias baixas nas subescalas da Escala Sócrates de "reconhecimento" e "ambivalência" e médias altas no que tange à tomada de decisão para a resolução do problema com drogas. Por isso, essa igualdade entre os grupos se destaca, pois a Ação e a Manutenção são tidos como estágios em que a negação do problema e a ambivalência sobre mudar o comportamento se dissiparam (Prochaska, 2013). Por outro lado, um estudo onde também fora aplicada a escala URICA, os resultados demonstraram que após a aplicação de um protocolo para aumentar a motivação o Short Motivational Programme (SMP), os infratores não transitaram entre os estágios, havendo inclusive regressões para estágios anteriores, o que gerou dúvidas a respeito da aplicação dos estágios de mudança para essa população (Yong, Williams, Provan, Clarke, \& Sinclair, 2015).

Já os dados da Régua de Prontidão demandam atenção uma vez que sendo um instrumento analógico-visual, demonstra de forma objetiva a opinião do participante e o quanto ele se acha preparado para efetuar sua mudança, sendo um dado motivacional subjetivo e rico. $\mathrm{Na}$ análise da Régua de Prontidão entre os Grupos, também se verificou não haver diferença estatisticamente significativa entre valores medianos ( $p=$ 0,083 ). Foram observadas em um estudo médias semelhantes na Régua de Prontidão em estudo com 395 usuários de crack, onde já se teorizava a alta correlação entre escores altos neste instrumento com o estágio de Ação, tendo resultados 


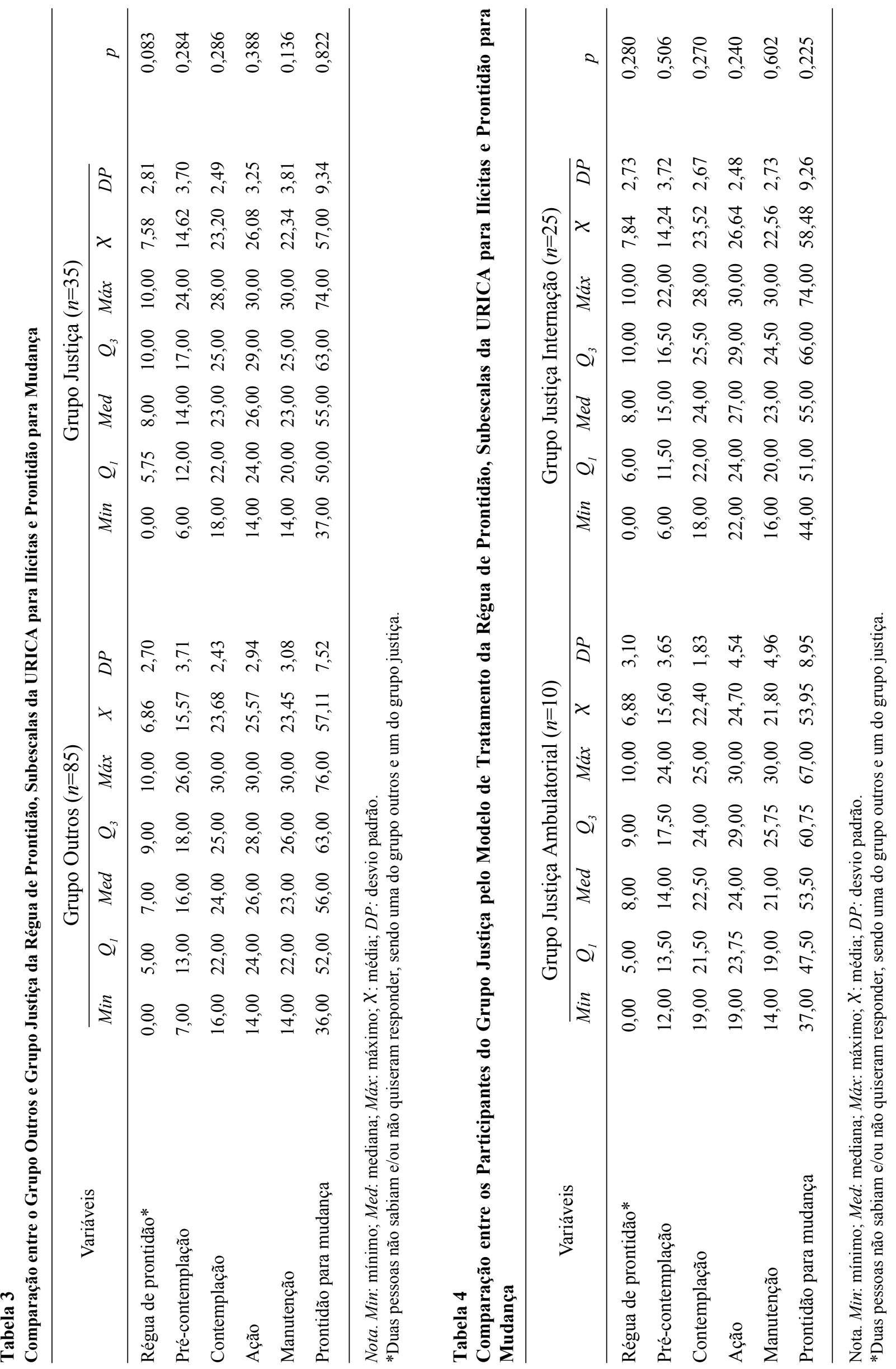


no tempo de abstinência (Szupszynski, Sartes, Andretta, \& Oliveira, 2014).

A respeito do número de participantes no Grupo Justiça $(n=35)$, essa quantidade correspondeu a quase todos os indivíduos encaminhados pela VEPEMA no segundo semestre do ano de 2015 e primeiro semestre de 2016. Assim, salienta-se que esse número ainda pequeno se deu pelo fato de o projeto Justiça Terapêutica ter iniciado em 2014 e se encontrava em sua fase piloto, não havendo, portanto, encaminhamentos em larga escala. Houve também três casos em que os encaminhados não foram localizados, todas no modelo ambulatorial que foram consideradas como perdas e, além disso, foram contabilizadas cinco recusas em participar da pesquisa: três do Grupo Justiça e duas pessoas do Grupo Outros. Das duas pessoas entre as três que se recusaram a participar do Grupo Justiça relataram que isso implicaria problemas com o Juiz e que só falariam sobre o encaminhamento com pessoas da justiça; a terceira pessoa relatou que estava indisposta a participar. As outras duas pessoas que se recusaram participar do Grupo Outros, apenas afirmaram que não gostariam de participar do estudo. A taxa de perdas ou recusas que correspondeu a $6,6 \%$ era esperada não comprometendo a análise comparativa pretendida e chamando a atenção quanto ao receio de algumas pessoas em falar sobre sua motivação.

Cabe ressaltar que o encaminhamento pela justiça se deu de forma voluntária e, às vezes, a pedido da própria pessoa. O projeto Justiça Terapêutica da VEPEMA baseia-se na expectativa de mudança de comportamento do indivíduo e nas novas políticas públicas de tratamento. Isso reflete a intensificação e a preocupação com a modificação de comportamento de uso de drogas na população carcerária nos últimos anos. Embora a maioria dos encaminhados tenha escolhido se tratar em CTs, uma pesquisa encomendada pela Secretaria Nacional de Políticas sobre Drogas (SENAD) e realizada pelo Instituto de Pesquisa Econômica Aplicada (IPEA) demonstrou que o encaminhamento pelo sistema de justiça é pequeno: apenas $9 \%$, sendo a família e por conta própria as maiores taxas de encaminhamento para tratamento em CTs (IPEA, 2016).
O perfil da amostra é de indivíduos com uso de múltiplas drogas, de maioria do sexo masculino e com prejuízos sociais e ocupacionais evidentes. Estudos referentes ao grupo de pessoas com problemas com a justiça corroboram estes dados, onde a baixa escolaridade, por serem de classes economicamente baixas, e o alto índice de desemprego desempenham uma dificuldade na reinserção social de pessoas com problemas judiciais (Oliveira \& Cardoso, 2004; Tavares, Scheffer, \& Almeida, 2012). Somente 24,2\% dos indivíduos foram classificados nas classes D-E, dessa forma pode-se considerar como a classe social menos favorecida somente a menor parte dos indivíduos que participaram desse estudo, ainda assim, com baixa escolaridade e alto índice de desemprego.

Comparando o número de mulheres em tratamento $(n=17 ; 14,2 \%)$ com o de homens ( $n=103 ; 85,8 \%$ ), pode-se pensar que esse fenômeno é também reflexo do restante do que acontece na maior parte do nosso país: pouca oferta de tratamento que contemple as vicissitudes das pessoas do sexo feminino como também há de incluir nessa realidade o despreparo técnico para esse tipo de população (Rennó et al., 2005). Apenas quatro mulheres foram encaminhadas para o tratamento pela justiça após serem presas por tráfico de drogas. O estudo de Lopes, de Mello e Argimon (2010) retratou a problemática de crimes e uso de drogas em mulheres presas, na qual a maioria da amostra possuía uma relação direta com as drogas, seja no uso ou na venda. Embora levantamentos indiquem o aumento de consumo e também do número de prisões relacionadas às drogas, como o tráfico (Laranjeira et al., 2012; Ministério da Justiça, 2015), esse tipo de benefício, o encaminhamento, ainda não é amplamente estendido para as mulheres.

Conforme observado na Tabela 4, não foram encontradas diferenças no Grupo Justiça entre os modelos de tratamento. Essa igualdade estatística demanda atenção, pois há um debate sobre a eficácia dos modelos de tratamento, porém no contexto deste estudo, as CTs foram mais procuradas do que o CAPS-AD pelos participantes do projeto Justiça Terapêutica. Os recursos terapêuticos ofertados em cada modelo de tratamento 
podem estar ligados a essa maior procura, e até influenciar na motivação. O sujeito pode esperar da internação uma solução rápida sobre o seu problema, assim ele se envolve de forma breve no tratamento, onde a laborterapia, o trabalho espiritual e religioso, a cooperação ou também pelo fato de estarem longe dos estímulos estressores ou eliciadores de fissura ou craving, contribuem para a maior procura. $\mathrm{O}$ tratamento em CTs democráticas é recomendado para aumentar a motivação visto que o encaminhado pela justiça pode adentrar ao tratamento sem motivação e, uma vez trabalhadas técnicas cognitivas e comportamentais, ter sucesso terapêutico (Stevens, 2013).

Em relação aos dias em abstinência na comparação entre os modelos de tratamento, fica nítido que no modelo ambulatorial (CAPS-AD), onde não se exige a abstinência, os sujeitos comumente mantêm o uso de álcool e outras drogas mesmo em situação de tratamento, o que pode influenciar no estágio de motivação. Cropsey et al. (2014) coloca que entre uma das tarefas para trabalhar e aumentar as taxas de motivação, voltadas para a abstinência, está o trabalho com as expectativas dos indivíduos dentro de programas de tratamento judicial. Nesse sentido, as expectativas dos encaminhados podem estar ligados ao desejo de ter sua ficha limpa perante a justiça.

Destacam-se os dados sobre os problemas associados ao uso, uma vez que na amostra total, $74,2 \%$ usavam drogas diariamente e $56,7 \%$ já cometeram algum pequeno delito para manter o consumo de drogas, principalmente o crack. Contudo, o estudo de Guimarães, Santos, Freitas e Araújo (2008) indicou não haver associação entre o padrão de consumo de crack e gravidade da dependência, quando analisados os antecedentes criminais de usuários de crack que estavam em desintoxicação em hospital psiquiátrico. O estudo de Tavares et al. (2012) constatou uma forte correlação entre uso de drogas, reincidência criminal e tipo de crime (roubos) com agressividade, onde a droga mais utilizada era o álcool. No presente estudo os participantes do Grupo Justiça relataram que os crimes mais prevalentes foram o tráfico de drogas $(42,9 \%)$ e o roubo $(25,7 \%)$.
Ainda sobre os dados acerca do uso de crack, mesmo não sendo a substância mais consumida na vida pelos participantes, ela foi responsável pela maioria dos casos de procura pelo tratamento $(50,8 \%)$, onde se inclui dezesseis pessoas do Grupo Justiça $(n=35)$. O estudo de Sousa, Ribeiro, Melo, Maciel e Oliveira (2013) avaliou com a Escala URICA os estágios de mudança em pacientes usuários e crack e de álcool internados em uma Comunidade Terapêtica e em um hospital, demonstrando que as pessoas em tratamento pelo uso de crack estavam mais motivadas que os usuários de álcool e que as pessoas internadas na CT também se encontravam mais motivadas que os internados no hospital.

Ao todo, dez participantes do Grupo Justiça relataram que não pensavam em se tratar antes de saberem que poderiam usufruir desse benefício, onde também colocaram informalmente para os pesquisadores que imaginavam serem obrigados a tal. O projeto Justiça Terapêutica não obriga os indivíduos a entrar e continuar no tratamento, ou seja, não os coage, diferentemente de programas de tratamento nos Estados Unidos. Sobre a coerção, ressalta-se que casos de estratégias de intervenção desse tipo podem até ser efetivos, contudo pesquisas demonstram que as mudanças duradouras acontecem quando o sujeito está verdadeiramente motivado para mudar seu comportamento (Farabee, Prendergast, \& Anglin, 1998). Além do mais, uma revisão sistemática de estudos sobre o tratamento compulsório não sugeriu evidências de que este modelo, no seu conjunto, apresente melhores resultados, sendo que alguns estudos sugeriram possíveis danos, como abusos de direitos humanos (Werb et al., 2016).

Nos dados obtidos se destaca o alto uso de sedativos no tratamento: $98 \%$ da amostra de 55 indivíduos fez uso por indicação médica, que fora receitados para Transtornos de Ansiedade, problemas para dormir, regularmente no início do tratamento em que ocorre a Síndrome de Abstinência. Uma pesquisa com presidiários na constatou uma alta prevalência de comorbidades psiquiátricas e de transtorno de personalidade associado ao Transtorno por Uso de Substâncias (Bennett \& Hunter, 2016). Ainda que os obje- 
tivos desse estudo não se aprofundem ao uso desses medicamentos, alerta-se que o uso de calmantes e sedativos por períodos longos tem a tendência de produzir dependência.

Apesar dos participantes não se encontrarem presos, algumas técnicas podem ser estudadas e postas em prática no contexto de tratamento fora da prisão para a Justiça Terapêutica, como o protocolo BSF (Beteende-Samtal-Förändring), traduzido como Comportamento-Conversa-Mudança, baseada na Entrevista Motivacional conjugada com cinco sessões semiestruturadas focadas no uso de substâncias e no comportamento criminal (Farbring \& Johnson, 2011). Os presos foram avaliados pela escala SOCRATES e após a intervenção as mudanças foram mais significativas na ambivalência e na ação, demonstrando assim que os passos para o aprimoramento de políticas públicas com presos usuários de drogas deve seguir modelos testados e que valorizem a motivação, podendo sustentar uma mudança mais duradoura.

Estes resultados demonstram importantes informações para pesquisadores, assim como para outros atores envolvidos no projeto de justiça terapêutica: poder judiciário, instituições de tratamento, apenados e seus familiares. A motivação é um importante componente presente na mudança de comportamento problemático e avaliá-la se faz fundamental em programas de encaminhamento como a Justiça Terapêutica. Nos últimos anos, o Brasil tem passado por diversos debates acerca do uso de drogas e seus efeitos na sociedade e nos índices de criminalidade, onde a consciência de se oferecer cada vez mais o tratamento em determinadas situações que envolvem as drogas, como no caso de pessoas com problemas com a justiça e a motivação é de suma importância para o sucesso de programas como tal.

A Justiça Terapêutica se constitui como uma medida alternativa que valoriza a saúde e essa coesão com a justiça visa não somente a coerção e a punição, mas sim que entre a punição no regime fechado e o tratamento, a segunda opção é a melhor, tendo o indivíduo possibilidades de solucionar seu problema com o uso de drogas e ainda cumprir sua pena, longe do ambiente prisional e dos riscos que a mesma oferece. Programas de encaminhamento judicial para tratamento para uso de drogas não são novos. Desde a década de 1990, estudos internacionais no sistema de justiça criminal já indicavam algumas práticas que devem ser implementadas em programas desse tipo, tais como períodos maiores de tratamento, acompanhamento no pós-tratamento, boa estrutura das instalações, flexibilidade do programa (caráter não autoritário) e fornecer assistência médica e capacitação para o trabalho (Anglin \& Hser, 1991). Logo, existem desafios a serem superados, como o despreparo das instituições que recebem os encaminhados, especialmente as CTs, que em alguns casos não contam com infraestrutura, recursos humanos qualificados ou até mesmo recursos financeiros.

Este estudo possui algumas limitações, como a alta disparidade entre o Grupo Justiça e o Grupo Outros, o que impossibilitou uma comparação estatística mais consistente. Contudo, trata-se de uma pesquisa feita com uma amostra que tende a aumentar, à medida que os resultados sobre o programa começarem a surgir. Outra limitação foi o fato do projeto de pesquisa não avaliar o estágio de motivação em adolescentes encaminhados. Apesar de no estado já haver um trabalho de encaminhamento de adolescentes para tratamento, ainda há a dificuldade de adesão ao tratamento e falta de estrutura para os atendimentos, contudo a Justiça Terapêutica é um projeto voltado para maiores de dezoito anos.

Espera-se que com o prosseguimento do projeto haja mais capacitações das equipes do poder judiciário e das instituições acolhedoras, buscando trabalhar mais os aspectos psicológicos e motivacionais, realizando a avaliação do estágio motivacional para a superação de possíveis resistências ao tratamento. Bem como, recomenda-se o aumento no número de pessoas encaminhadas entre os gêneros, abarcando mais indivíduos sem condições de ter seus direitos garantidos por advogados. Com a crescente demanda por tratamento, sugere-se investir na continuidade de estudos que abarquem os estágios de motivação com essa população no Brasil, inclusive com amostra maiores e melhores condições de representatividade, que nessa ocasião, em virtude do número ainda pequeno de sujeitos 
encaminhados, não foi possível realizar. Por fim, faz-se necessária a realização de estudos de seguimento com tal população encaminhada, a fim de verificar em longo prazo os resultados dessa importante política pública.

\section{Contribuição dos autores}

Contribuição substancial no conceito e desenho do estudo: Itamar José Félix Junior e Paulo Renato Vitória Calheiros.

Contribuição para a coleta de dados: Itamar José Félix Junior.

Contribuição para análise e interpretação de dados: Itamar José Félix Junior, Paulo Renato Vitória Calheiros e Pedro di Tárique Barreto Crispim.

Contribuição para a preparação do manuscrito: Itamar José Félix Junior, Paulo Renato Vitória Calheiros e Pedro di Tárique Barreto Crispim.

Contribuição para revisão crítica, adicionando conteúdo intelectual: Itamar José Félix Junior, Paulo Renato Vitória Calheiros e Pedro di Tárique Barreto Crispim.

\section{Conflitos de interesse}

Os autores declaram não ter conflito de interesse relacionado à publicação deste manuscrito.

\section{Referências}

Associação Brasileira de Empresas de Pesquisa. (2015). Critério de classificação econômica Brasil 2015. Recuperado em http://www.abep. org/criterioBrasil.aspx

Anglin, M. D., \& Hser, Y. I. (1991). Criminal justice and the drug-abusing offender: Policy issues of coerced treatment. Behavioral Sciences \& The Law, 9(3), 243-267. doi: 10.1002/ bsl.2370090304

Bertolucci, P. H., Brucki, S., Campacci, S. R., \& Juliano, Y. (1994). O mini-exame do estado mental em uma populaçäo geral: Impacto da escolaridade. Arquivos de Neuro-Psiquiatria, 52(1), 1-7. doi: 10.1590/S0004-282X1994000100001

Bennett, A., \& Hunter, M. (2016). Implementing evidence-based psychological substance misuse interventions in a high secure prison based personality disorder treatment service. Advances in Dual Diagnosis, 9(2-3), 108-116. doi: 10.1108/ ADD-02-2016-0002

Cropsey, K. L., Leventhal, A. M., Stevens, E. N., Trent, L. R., Clark, C. B., Lahti, A. C., \& Hendricks, P. S. (2014). Expectancies for the effectiveness of different tobacco interventions account for racial and gender differences in motivation to quit and abstinence self-efficacy. Nicotine \& Tobacco Research, 16(9), 1174-1182. doi: $10.1093 / \mathrm{ntr} / \mathrm{ntu} 048$

El-Bassel, N., Schilling, R. F., Ivanoff, A., Chen, D. R., Hanson, M., \& Bidassie, B. (1998). Stages of change profiles among incarcerated drug-using women. Addictive Behaviors, 23(3), 389-394

Farbring, C. A.., \& Johnson, W. R. (2011). Entrevista Motivacional no Sistema Prisional - Uma tentativa de implementar a Entrevista Motivacional na Justiça Criminal. In H. Arkowitz, H. A. Westra, W. R. Miller, \& S. Rollnick, Entrevista Motivacional no tratamento de problemas psicológicos (pp. 253-267). São Paulo, SP: Roca.

Farabee, D., Prendergast, M., \& Anglin, M. D. (1998). The Effectiveness of Coerced Treatment for Drug-Abusing Offenders. Federal Probation, 62(1), 3-10. Retrieved from http://heinonline. org/HOL/LandingPage?handle=hein.journals/ fedpro62\&div $=5 \&$ id $=\&$ page $=$

Guimarães, C. F., Santos, D. D., Freitas, R. D., \& Araújo, R. B. (2008). Perfil do usuário de crack e fatores relacionados à criminalidade em unidade de internação para desintoxicação no Hospital Psiquiátrico São Pedro de Porto Alegre (RS). Revista de Psiquiatria do Rio Grande do Sul, 30(2), 101-108. doi: 10.1590/S010181082008000300005

Instituto de Pesquisa Econômica Aplicada. (2016). Nota Técnica Perfil das Comunidades Terapêuticas Brasileiras. Recuperado em http://www. ipea.gov.br/portal/index.php?option=com_cont ent\&view $=$ article $\&$ id $=29865 \&$ Itemid $=6$

Kelly, J. F., Finney, J. W., \& Moos, R. (2005). Substance use disorder patients who are mandated to treatment: Characteristics, treatment process, and 1-and 5-year outcomes. Journal of Substance Abuse Treatment, 28(3), 213-223. doi: 10.1016/j.jsat.2004.10.014

Laranjeira, R., Madruga, C. S., Ribeiro, M., Pinsky, I., Caetano, R., \& Mitsuhiro, S. S. (2012). II 
LENAD-Levantamento Nacional de Álcool e Drogas. São Paulo, SP: Instituto Nacional de Ciência e Tecnologia para Políticas Públicas do Álcool e Outras Drogas.

Lei $\mathrm{n}^{\mathrm{o}}$ 11.343, de 23 de agosto de 2006. (2006, 24 ago.). Institui o Sistema Nacional de Políticas Públicas sobre Drogas - Sisnad; prescreve medidas para prevenção do uso indevido, atenção e reinserção social de usuários e dependentes de drogas; estabelece normas para repressão à produção não autorizada e ao tráfico ilícito de drogas; define crimes e dá outras providências. Diário Oficial da União. Recuperado em http://www.planalto.gov.br/ccivil_03/_ato20042006/2006/lei/111343.htm

Lopes, R. M., de Mello, D. C., \& Argimon, I. I. (2010). Mulheres encarceradas e fatores associados a drogas e crimes. Ciências \& Cognição, 15(2), 121-131. Recuperado em http://pepsic.bvsalud. org/scielo.php?script=sci_arttext\&pid $=\mathrm{S} 180658$ 212010000200011\&lng=pt\&tlng=pt

Ministério da Justiça. (2015). Levantamento Nacional de Informações Penitenciárias-Infopen Mulheres 2014. Recuperado em https://www. justica.gov.br/noticias/estudo-traca-perfil-da-populacao-penitenciaria feminina-no-brasil/ relatorio-infopen-mulheres.pdf

Oliveira, H. B. D., \& Cardoso, J. C. (2004). Tuberculose no sistema prisional de Campinas, São Paulo, Brasil. Revista Panamericana de Salud Pública, 15(3), 185-193. doi: 10.1590/S102049892004000300008

Oliveira, M. D. S., Ludwig, M. W. B., Freire, S., \& Zanetello, L. B. (2012). O modelo transteórico de mudança no enfoque grupal. In I. Andretta \& M. D. S. Oliveira (Eds.), Manual Prático de Terapia Cognitivo-Comportamental (pp. 455466). São Paulo, SP: Casa do Psicólogo.

Oliveira, M. D. S., Szupszynski, K. P. D. R., \& DiClemente, C. (2010). Estudo dos estágios motivacionais no tratamento de adolescentes usuários de substâncias psicoativas ilícitas. Psico, 41(1). Recuperado em http://revistaseletronicas.pucrs.br/ojs/index.php/revistapsico/article/ view/7207/0

Polaschek, D. L., Anstiss, B., \& Wilson, M. (2010). The assessment of offending-related stage of change in offenders: Psychometric validation of the URICA with male prisoners. Psychology, Crime \& Law, 16(4), 305-325. doi: $10.1080 / 10683160802698766$
Prendergast, M., Greenwell, L., Farabee, D., \& Hser, Y. I. (2009). Influence of perceived coercion and motivation on treatment completion and rearrest among substance-abusing offenders. The Journal of Behavioral Health Services \& Research, 36(2), 159-176. doi: 10.1007/s11414008-9117-3

Prochaska, J. O. (2013). Transtheoretical model of behavior change. In Encyclopedia of behavioral medicine (pp. 1997-2000). New York: Springer.

Prochaska, J. O., DiClemente, C. C., \& Norcross, J. C. (1992). In search of how people change: Applications to addictive behaviors. American Psychologist, 47, 1102-1114. doi: 10.1037/0003066X.47.9.1102

Rennó, J., Jr., Fernandes, C. E., Mantese, J. C., Valadares, G. C., Fonseca, Â. M., Diegoli, M., ...Hochgraf, P. (2005). Saúde mental da mulher no Brasil: Desafios clínicos e perspectivas em pesquisa. Revista Brasileira de Psiquiatria, 27(Supl. 2), S73-S76. Recuperado em http://www.scielo. br/pdf/rbp/v27s2/pt_a07v27s2.pdf

Serafini, K., Stewart, D. G., Wendt, D. C., \& Donovan, D. M. (2017). Perceived parental support and adolescent motivation for substance use change: A preliminary investigation. Addiction Research \& Theory, 1-6. doi: 10.1080/16066359.2017.1342819

Soares, M. M., Filho, \& Bueno, P. M. M. G. (2016). Demografia, vulnerabilidades e direito à saúde da população prisional brasileira. Ciência \& Saúde Coletiva, 21(7), 1999-2010. doi: 10.1590/141381232015217.24102015

Sousa, P. F., Ribeiro, L. C. M., Melo, J. R. F. D., Maciel, S. C., \& Oliveira, M. X. (2013). Dependentes químicos em tratamento: Um estudo sobre a motivação para mudança. Temas em Psicologia, 21(1), 259-268. doi: 10.9788/TP2013.1-18

Stevens, A. (2013). Prisoners' motivations for therapeutic community treatment In search of a 'different'approach to offender rehabilitation. Probation journal, 60(2), 152-167. doi: $10.1177 / 0264550513478321$

Szupszynski, K. P. D. R., \& Oliveira, M. D. S. (2008). Adaptação brasileira da University of Rhode Island Change Assessment (URICA) para usuários de substâncias ilícitas. PsicoUSF, 13(1), 31-39. Recuperado em http://pepsic.bvsalud.org/scielo. php?script $=$ sci_arttext\&pid $=$ S 14138271200800 0100005\&lng=pt\&tlng=pt 
Szupszynski, K. P. D. R., Sartes, L. M. A., Andretta, I., \& Oliveira, M. D. S. (2014). Processos de mudança cognitivos e comportamentais em usuários de crack em tratamento. Revista Brasileira de Terapias Cognitivas, 10(1), 11-18. doi: https://dx.doi.org/10.5935/1808-5687.20140003

Tavares, G. P., Scheffer, M., \& Almeida, R. M. M. D. (2012). Drogas, violência e aspectos emocionais em apenados. Psicologia: Reflexão e Crítica, 25(1), 89-95. Recuperado em http://www. scielo.br/pdf/prc/v25n1/a11v25n1

Velasquez, M., Maurer, G., Crouch, C., \& DiClemente, C. (2001). Group treatment for substance abuse: A stages-of- change therapy manual. New York: The Guilford Press.

Werb, D., Kamarulzaman, A., Meacham, M. C., Rafful, C., Fischer, B., Strathdee, S. A., \& Wood, E. (2016). The effectiveness of compulsory drug treatment: A systematic review. International
Journal of Drug Policy, 28, 1-9. doi: 10.1016/j. drugpo.2015.12.005

Wilson, D. B., Mitchell, O., \& MacKenzie, D. L. (2006). A systematic review of drug court effects on recidivism. Journal of Experimental Criminology, 2(4), 459-487. doi: 10.1007/ s11292-006-9019-4

Yong, A. D., Williams, M. W. M., Provan, H., Clarke, D., \& Sinclair, G. (2015). How do offenders move through the stages of change? Psychology, Crime \& Law, 21(4), 375-397. doi: 10.1080/1068316X.2014.989166

Recebido: 22/06/2017

$1^{a}$ revisão: $17 / 08 / 2017$

Aceite final: $22 / 08 / 2017$

(cc)BY (C) O(s) autor(es), 2018. Acesso aberto. Este artigo está distribuído nos termos da Licença Internacional Creative Commons Atribuição 4.0 (http://creativecommons.org/licenses/by/4.0/), que permite o uso, distribuição e reprodução sem restrições em qualquer meio, desde que você dê crédito apropriado ao(s) autor(es) original(ais) e à fonte, fornecer um link para a licença Creative Commons e indicar se as alterações foram feitas. 